\title{
COMPARAÇÃO DAS PARASITOSES MAIS ENCONTRADAS EM DOIS MUNICÍPIOS DA REGIÃO SUL DE GOIÁS
}

\author{
Islaine do Prado SANTOS ${ }^{1}$ \\ Roberta Mendes Ferreira ISAAC ${ }^{2}$ \\ 1: Discente do curso de Enfermagem da Faculdade de Filosofia e Ciências Humanas de Goiatuba. \\ E-mail: i.santos@outlook.com.br \\ 2: Docente do Curso de Enfermagem da Faculdade de Filosofia e Ciências Humanas de Goiatuba. \\ E-mail: robertaatbg@ hotmail.com
}

Recebido em: 19/04/2015 - Aprovado em: 26/08/2015 - Disponibilizado em: 30/10/2015

\begin{abstract}
Resumo: As parasitoses intestinais constituem um sério problema de saúde pública, especialmente nos países tropicais e subtropicais. São muito prevalentes em famílias urbanas e rurais de baixa renda, nas quais as deficiências nutricionais são também mais frequentes. O presente estudo teve como objetivo comparar e identificar as principais parasitoses encontradas em análises laboratoriais em Cromíniae em Morrinhos - Goiás, tendo em vista à diferença socioeconômica da população e o acesso à saúde básica. Todas as amostras analisadas foram submetidas aos exames coproparasitológicos de sedimentação espontânea, segundo o método desenvolvido por Hoffman, Pons e Janer (1934). Os resultados mostraram uma grande incidência de Endolimax nana em ambos os municípios, a grande prevalência de parasitoses intestinais encontradas nesse tipo de estudo, pode servir de alerta para as autoridades e o poder público, para que medidas urgentes sejam tomadas no sentido da erradicação do problema, ou as altas taxas dos parasitos encontrados podem servir para classificá-los como sendo doenças negligenciadas pela saúde pública e pelos órgãos responsáveis pela formulação de políticas de saúde coletiva nos municípios estudados.
\end{abstract}

Palavras-chave: Parasitoses. Saúde Pública. Parasitos intestinais. Saúde Coletiva. Saúde Básica.

\section{COMPARITION OF PARASITOSIS MOST FOUND IN TWO CITIES OF THE REGION SOUTH OF GOIÁS}

Abstract: Intestinal parasitic are a serious public health problem, especially in tropical and subtropical countries. They are very prevalent in urban and rural low-income families in which nutritional deficiencies are also more frequent. This study aimed to compare and identify the main parasites found in laboratory tests in Cromínia and Morrinhos - Goiás, given the socioeconomic differences of the population and access to basic health. All samples were submitted to fecal examinations of spontaneous sedimentation, according to the method developed by Hoffman, Pons and Janer (1934). The results showed a high incidence of Endolimax nana in both municipalities, the high prevalence of intestinal parasites found in this type of study can serve as a warning to the authorities and the government, so that urgent steps be taken towards the eradication of the problem or high rates of parasites found can be used to classify them as neglected diseases for public health and bodies responsible for the formulation of public health policies in the cities studied.

Key-words: Parasitosis. Public Health. Intestinal parasites. Colletive Health. Basic Health.

\section{INTRODUÇÃO}

Segundo NEVES (2005) o

parasitismo é uma associação entre os seres

vivos, na qual somente um deles é

beneficiado na relação, nesse caso o

hospedeiro sofre constante espoliação,

fornecendo alimento e abrigo para o parasito.

Tal tipo de relação tende ao equilíbrio, pois seria prejudicial ao parasito a morte de seu hospedeiro. Em zonas endêmicas de certas parasitoses, como a malária, por exemplo, a letalidade da população autóctone é muito baixa, entretanto quando pessoas de fora entram nessa área, adquirem essa doença na forma mais patogênica.

As parasitoses intestinais constituem um sério problema de saúde pública, 
especialmente nos países tropicais e subtropicais. São muito prevalentes em famílias urbanas e rurais de baixa renda, nas quais as deficiências nutricionais são também mais frequentes. Assim, nessa numerosa população, as repercussões das parasitoses intestinais somam-se aos efeitos das carências socioeconômicas e ambientais (TASHIMA et al., 2005). A maioria das pessoas portadoras de parasitas intestinais apresentam intensos distúrbios orgânicos, podendo levar muitas vezes à incapacitação dos indivíduos infectados. $\mathrm{Na}$ maioria das vezes correspondem ao único processo mórbido do paciente, outras vezes vêm agravar outras patologias concomitantes (CIMERMAN et al., 2001).

As condições de saúde em seu contexto de prevenção, manutenção e tratamento das enfermidades no Brasil e em outros países economicamente desfavoráveis, são graves problemas (MARQUES et al, 2005), assim como são escassas as informações sobre parasitoses (CARVALHO et al, 2002) ou mesmo inexistentes para que providências sejam tomadas. As parasitoses sofrem variações inter e intrarregionais, dependendo das condições sanitárias, educacionais, sociais, índice de aglomeração de pessoas, condições de uso e contaminação do solo, da água e alimentos, e da capacidade de evolução das larvas e ovos de helmintos e de cistos de protozoários em cada uma dessas localidades (MARQUES et al, 2005). O presente estudo teve como objetivo comparar e identificar as principais parasitoses encontradas em análises laboratoriais em duas cidades, Cromínia e Morrinhos, ambos municípios localizados na Região Sul do Estado de Goiás, tendo em vista a diferença socioeconômica da população e o acesso a saúde básica.

\section{REVISÃO DE LITERATURA}

Segundo FERREIRA et al, (2006), as infecções parasitárias podem ser definidas como, a penetração e desenvolvimento, ou também a multiplicação de um agente infeccioso no homem ou animal. Estima-se que o ser humano possa ser infectado por mais de 100 tipos de parasitas ao longo de sua vida, sendo que estas infecções parasitárias possam ser responsáveis por mais de um milhão de mortes por ano. Decorrentes da presença de macroparasitas (helmintos) e/ou microparasitas (protozoários) no intestino que comprometem cerca de $25 \%$ da população mundial (FELDMAN, et al., 2002).

As helmintíases dividem-se em: nematelmintos (vermes de corpo cilíndrico) e platelmintos (vermes de corpo achatado dorso-ventralmente), cestóides (forma de fita e segmentados) ou trematóides (forma de folha e não segmentados). A prevalência varia conforme a região analisada, e depende dos aspectos climáticos, das características do solo, dos hábitos alimentares e de higiene e 
das condições sanitárias dos indivíduos. A invasão do parasita ocorre através da pele (pelo contato direto com o solo contaminado) e/ou pela boca (ingestão de água e/ou alimentos contaminados, fomites, perversão do apetite ou prática sexual) (GASPARINI, 2005).

De acordo com PEDRAZZANI et al, (1988) as parasitoses intestinais constituem um grave problema de saúde pública além de contribuir para problemas sociais, financeiros, econômicos e médicos sobretudo nos países em desenvolvimento. As doenças parasitárias são importantes pela mortalidade que os procede e pela frequência com que produzem déficits orgânicos, sendo que se tornam um dos principais fatores debilitantes da população, principalmente quando se associam frequentemente a quadros de diarreia crônica e desnutrição, comprometendo assim, nas faixas etárias mais jovens da população, o seu desenvolvimento físico e intelectual, (SALATA et al., 1972).

A prevalência de infecções por parasitas intestinais é um dos indicadores confiáveis do status socioeconômico de uma população (ASTAL, 2004). Pode estar associada a diversos fatores determinantes, como instalações sanitárias muitas vezes inadequadas, poluição fecal da água e dos alimentos consumidos, fatores socioculturais, contato com animais, ausência de saneamento básico, além da idade do hospedeiro e do tipo de parasita infectante (GAMBOA, et al., 2003).

No Brasil, apesar da diferença entre as regiões, a contaminação do meio ambiente é intensa, com elevada prevalência das parasitoses intestinais e associação dos vários parasitas. A maioria dos pacientes é assintomática ou oligossintomática, entretanto alguns podem apresentar quadros clínicos graves, o que demonstra o irregular padrão evolutivo das parasitoses (FELDMAN et al., 2002). As manifestações clínicas podem ou não estar presentes, variando de ausência de sintomas a estado subagudo ou crônico (MILLER, et al., 2003). Os sintomas, muitas vezes, são vagos e inespecíficos, o que dificulta os diagnósticos clínicosespecíficos. Podem manifestar-se por diarreia (aquosa, mucóide, aguda, persistente e intermitente), dor abdominal (desconforto vago à cólicas), dispepsia, anorexia, astenia, emagrecimento e distensão abdominal (YERELI, et al., 2004).

No Brasil, a frequência de infecção por enteroparasitas varia de acordo com a região estudada e com a população que em geral não são significativamente elevadas se forem comparadas com às da população de faixa etária de 0-12 anos (SATURNINO et al., 2003). Segundos os autores, na literatura, existem diversos trabalhos de estudos epidemiológicos na população dos municípios em diversos estados brasileiros, onde os helmintos registrados parasitando os humanos podemos destacar o Ascaris lumbricoides, 
Trichuristrichiura, Strongyloidesstercoralis, Enterobius vermiculares, ancilostomídeos, Hymenolepis nana, Taenia sp. e Shistosoma mansoni, como mais abundantes respectivamente.

Em exames coproparasitológicos realizados em laboratórios especializados frequentemente encontram Entamoeba coli $e$ Endolimax nana, entretanto, são considerados comensais,normalmente não tendo ação patogênica nos hospedeiros (FERREIRA; ANDRADE, 2005).

Os helmintos e protozoários ao terem seus ovos, larvas ou cistos eliminados junto comas fezes do hospedeiro, contaminam o ambiente e o solo, podendo os ovos ou cistos serem levados aosalimentos, ou serem arrastados pela água. Em relação à água, a contaminação pode ocorrer das seguintes formas: porenxurradas que atingem mananciais que são utilizados no abastecimento de cidades, sendo que em algumas localidades a água não é tratada corretamente, e nairrigação de plantações, cujos produtores não fazem o tratamento da água, sendo que muitas vezes ela vem diretamente do córrego para a plantação, e através do contato da mão suja com a boca, que ocorre tanto em adultoscomo em crianças, além da penetração das larvas através da pele (PEDROSO \&SIQUEIRA, 1997).

Sendo assim, fatores ambientais podem favorecer o desenvolvimento e a propagação dasformas infectantes dos parasitas, enquanto o nível socioeconômico entre outros fatores são responsáveis pela contaminaçãodo ambiente com esses parasitas e, consequentemente, do hospedeiro (PEDROSO \& SIQUEIRA, 1997). Tal problematem como principais afetados as populações de baixa renda, que vivem emcondições precárias de saneamento básico e higiene, sendo as crianças as maisafetadas (FERREIRA et al., 2006).

\section{MATERIAL E MÉTODOS}

Foram analisados resultados de exames parasitológicos de fezes, realizados no Laboratório Municipal de Cromínia, na cidade de Cromínia e em um Laboratório Particular na cidade de Morrinhos, no Estado de Goiás, compreendendo o período dos meses de Setembro e Outubro de 2013. Foram estudados 58 amostras de 58 pacientes diferentes em Cromínia e 60 amostras de 60 pacientes diferentes na cidade de Morrinhos. As amostras eram recebidas de acordo com os pedidos médicos, portanto os pacientes não se queixavam exclusivamente de parasitoses intestinais, foram avaliadas apenas as amostras positivas para algum tipo de parasita, sendo que as negativas não foram citadas.

O material foi coletado pelo próprio paciente em recipiente limpo e seco, com boca, larga com capacidadeaproximada de $250 \mathrm{ml}$, possuindo mecanismo de 
vedaçãohermética, a qual impede o derrame e preserva a umidade. Os pacientes foram orientados, caso não conseguissem coletar as fezes no dia do exame, a coletar no dia anterior e armazenar em refrigerador, com temperaturas entre 4 e $5^{\circ} \mathrm{C}$, por não mais que 24 horas.

Todas as amostras analisadas foram submetidas aos exames coproparasitológicosde sedimentação espontânea, segundo o método desenvolvido por Hoffman, Pons e Janer (LUDWIG et al., 1999), método muito utilizado por possuir precisão, apresentar resultados mais claros e facilitar o diagnóstico das parasitoses presentes no material que está sendo analisado (TIBIRIÇÁ et al., 2009).

As fezes frescas contidas no recipiente de coleta foram pesadas e em seguida homogeneizadas com água comum no próprio recipiente, foram retirados do material fecal entre 2 a $4 \mathrm{~g}$, que foram homogeneizadas com água e passadas para um cálice de sedimentação de $100 \mathrm{ml}$, através de uma gaze dobrada em quatro partes, e seu volume é completado com água. Esta suspensão é deixada em repouso para que ocorra uma sedimentação espontânea das estruturas parasitárias e então, após 4 a 6 horas, o material é examinado ao microscópioem objetivas de $10 \mathrm{X}$ e $40 \mathrm{X}$ (REY, 2002).
O Método desenvolvido por Hoffman, Pons e Janer (1934), também conhecido como método da sedimentação espontânea, ou HPJ, é comumente aplicado na rotina laboratorial ou inquéritos coproparasitológicos, pois possui complexidade e custo baixos na sua execução (TIBIRIÇÁ et al., 2009). Como salientado por Araújo et al. (2003), fatores como volume do material examinado, número de ovos produzidos pelo parasito e carga parasitária podem influenciar no diagnóstico pelo método HPJ.

\section{RESULTADOS E DISCUSSÃO}

As enteroparasitores constituem-se em problemas de sérios para os agentes responsáveis pela saúde pública, por serem de fácil transmissão e resistente a vários ambientes, podendo infestar populações inteiras que fiquem suscetíveis a esses parasitos. Na Tabela 1 podem ser observados os principais parasitas encontrados no município de Cromínia, o que pode observado é que nos meses de análise, os parasitos Endolimax nana e Entamoeba coli, foram os principais casos encontrados, sendo que $E$. coli com maior presença no mês de setembro e E. histolytica no mês de outubro. 
Tabela 1 - Parasitas encontrados em Cromínia-GO.

\begin{tabular}{ccccc}
\hline Parasitas & Agosto & Setembro & Outubro & Novembro \\
\hline Ancyslostomaduodenale & - & 1 & - & - \\
Endolimax nana & - & 12 & 2 & - \\
Entamoeba coli & - & - & 2 & - \\
Entamoebahistolytica & - & 1 & 6 & - \\
Giardialamblia & - & 1 & 1 & - \\
Hymenolepis nana & - & 1 & - & - \\
Iodamoebabutschllii & - & 2 & - & - \\
\hline
\end{tabular}

Fonte: Dados da pesquisa.

Nos meses de Agosto e Novembro não houve análises no laboratório Municipal de Cromínia, dificultando a coleta de dados para a presente pesquisa. Que pode ser observado na Tabela 1 é que foram encontrados 6 casos, da população selecionada, nos exames realizados no laboratório municipal da cidade de Cromínia. Não foi possível identificar a faixa etária do grupo que apresentou esses casos de infestação, por questões de sigilo laboratorial dos pacientes. Um dos fatores que podem ser associados à tamanha infestação de $E$. histolytica são as condições sócio-econômicas precárias das populações analisadas e a falta de saneamento básico, pouco utilizado na região Sul do Estado de Goiás, mas como destacado por Silva et al. (2005), dependendo da população estudada os métodos coproscópicos podem subestimar ou superestimar a prevalência da doença, sendo indicado o tratamento para evitar os possíveis sintomas que o parasito pode causar no organismo do hospedeiro.

Com relação ao mono e ao poliparasitismo, a distribuição das espécies entre os grupos analisados no presente trabalho não mostrou diferença, o mesmo acontecendo com cada espécie analisada. Quantos aos protozoários intestinais, analisando a E. histolytica em analises, grande parte dos cistos encontrados apresentam até quatro núcleos, o presente trabalho não fez a diferenciação morfométrica dos cistos encontrados nas amostras analisadas.

A alta frequência de exames negativos observados durante a condução do trabalho pode estar associada ao ciclo reprodutivo dos parasitos, pois não houve repetições de coletas com a população utilizadas nas duas cidades. A alta infestação de por E. nana, está de acordo com os resultados encontrados por Ferreira e Vieira (2006), apesar desse parasita não apresentar danos ao seu hospedeiro, a presença dele está relacionada com condições de insalubres de higiene, tanto pessoal como ambiental, indicando, principalmente, a transmissão fecal-oral, que ocorre, frequentemente, por meio de fonte de água não tratadas da maneira correta ou sem tratamento algum. A Figura 1 
apresenta a dispersão da população dos mais fácil sua visualização. parasitos nos meses analisados, o que torna

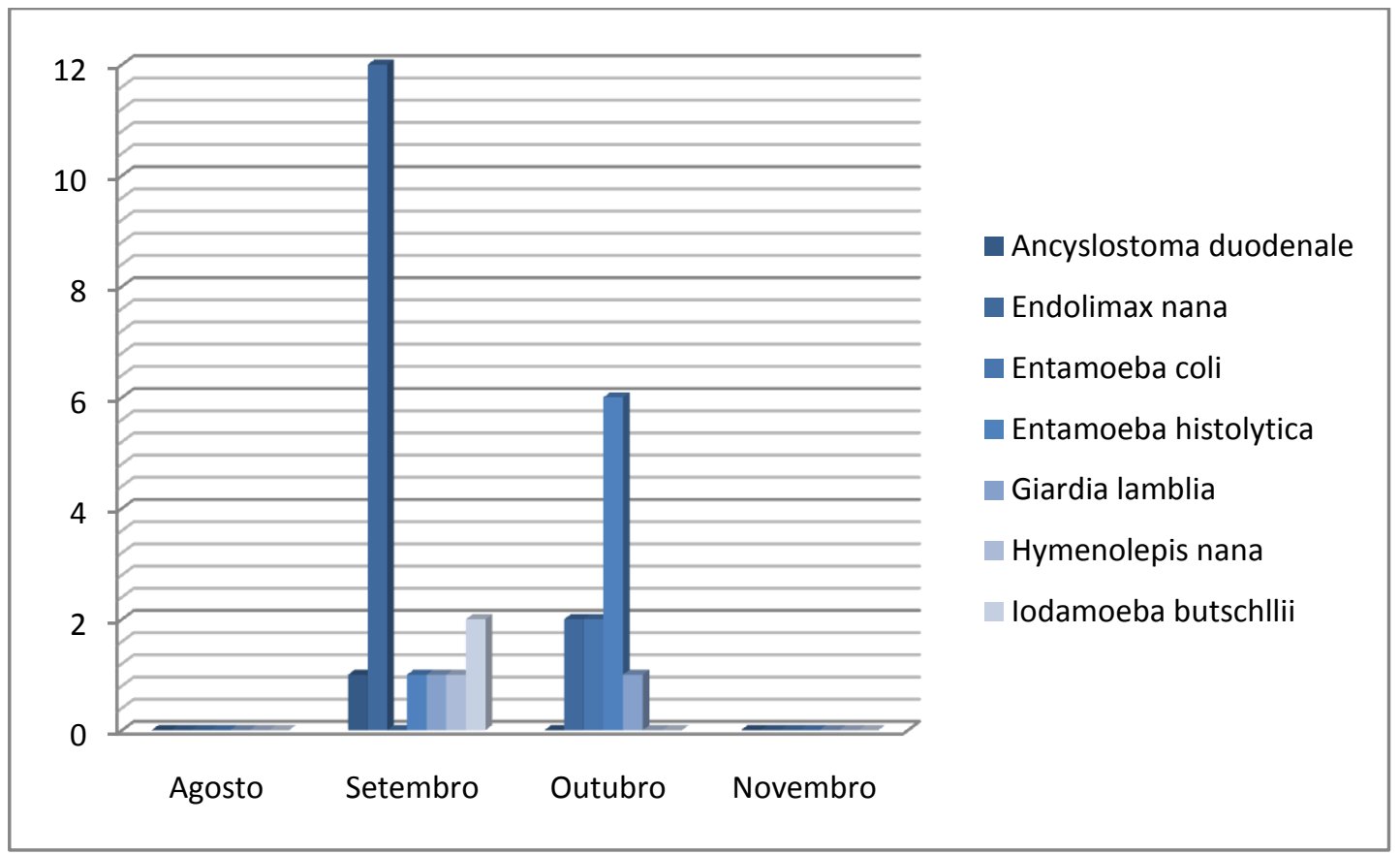

Figura 1 - Casos de parasitas encontrados na cidade de Cromínia-GO nos meses de Setembro e Outubro de 2013.

Amebas são parasitos sarcodinos com fina película proteica revestindo externamente acélula provida basicamente de vacúolos e núcleo. Os inúmeros pseudópodos, usados paralocomoção e captura de partículas nutrientes (endocitose), fornecem às amebas uma formairregular e assimétrica.Podem ser encontradas em algumas cavidades do corpo humano, por exemplo, intestinogrosso (Entamoebahistolytica e E. coli) e cavidade oral (E. gingivalis), mas a única espécieintestinal patogênica é a Entamoebahistolytica (SANTOS; SOARES, 2008).

Além dela, uma infinidade de outras amebascomensais, não parasitas e não patogênicas, pode ser encontradas nestas cavidades humanas e,no caso das amebas comensais intestinais, sua presença é indicativa de maus hábitos de higiene.Entre as espécies de amebas comensais intestinais existem sarcodinos muito semelhantesà $E$. histolytica e que podem gerar diagnósticos falso positivos se o técnico que as observa não forbem treinado. Entre elas temos: Entamoebadispar, morfologicamente idêntica à E. histolytica, E. coli, Iodamoebabutschlii, Endolimax nana, dentre outras.Em relação à E. histolyticaos mecanismos envolvidos na patogênese incluem moléculas da superfície da ameba queaderem a membrana do enterócito e de outras células, inclusive leucócitos, inserção de um poro,o amebaporo, na membrana da célula hospedeira, levando-a 
à lise osmótica (entrada de água) ea secreção de proteases capazes de digerir a membrana basal (substância cimentante queassenta o epitélio sobre o conjuntivo) (SANTOS; SOARES, 2008).

A Tabela 2 apresenta os dados encontrados para a cidade de Morrinhos. Os dados não diferem muitos dos encontrados para a cidade de Cromínia, pois foram encontradas E. nana e E. coli, mas nesse município as análises conseguiram captar a presença de Giardialamblia, parasita que não apresentou grande importância nas análises realizadas em

Cromínia.

Tabela 2 - Parasitas encontradas em Morrinhos-GO

\begin{tabular}{lllll}
\hline Parasitas & Agosto & Setembro & Outubro & Novembro \\
\hline Endolimax nana & 9 & 12 & 18 & 16 \\
Entamoeba coli & 9 & 6 & 4 & 8 \\
Giardialamblia & 6 & 9 & 4 & 8 \\
\hline
\end{tabular}

Fonte: Dados da pesquisa.

Nota-se que, em relação às outras parasitoses encontradas Endolimax nana, teve prevalência maior. Entamoeba coli e Giardialambliaobtiveram o mesmo número de casos positivos, entretanto $E$. coli não se trata de um parasita comensal. A Figura 2 torna mais fácil a visualização da dispersão da população dos parasitos encontrados no presente estudo.

A doença relacionada à infestação de G. lambliarecebe o nome de giardíase. Contrai-se giardíase ao ingerir cistos, provenientes de fezes humanas (e/ou de alguns animais, principalmente animais domésticos, como o cachorro) contaminantes de água não tratada e alimentos de origem vegetal higienizados inadequadamente. O cisto sofre ação do suco gástrico e entérico liberando seu conteúdo que finaliza a divisão celular e dá origem aos trofozoítos que colonizarão e formarão o tapete sobre a mucosa. Ao seguirem junto ao bolo alimentar que sofrerá a desidratação no trânsito pelo intestino grosso, o trofozoíto passa pelo processo de encistamento, de modo que, em fezes formadas, serão eliminados cistos de giárdia. Entretanto, não se sabe ao certo o porquê, durante alguns períodos com duração de cerca de dez dias, o trofozoíto perde ou suspende a capacidade de se encistar. Os trofozoítos que por ventura ali estiveram, serão destruídos durante $\mathrm{o}$ processo de formação do bolo fecal. Por isso, a este período de eliminação de fezes formadas, sem a presença de cistos, chamamos de período negativo

(NEVES,

2005). 


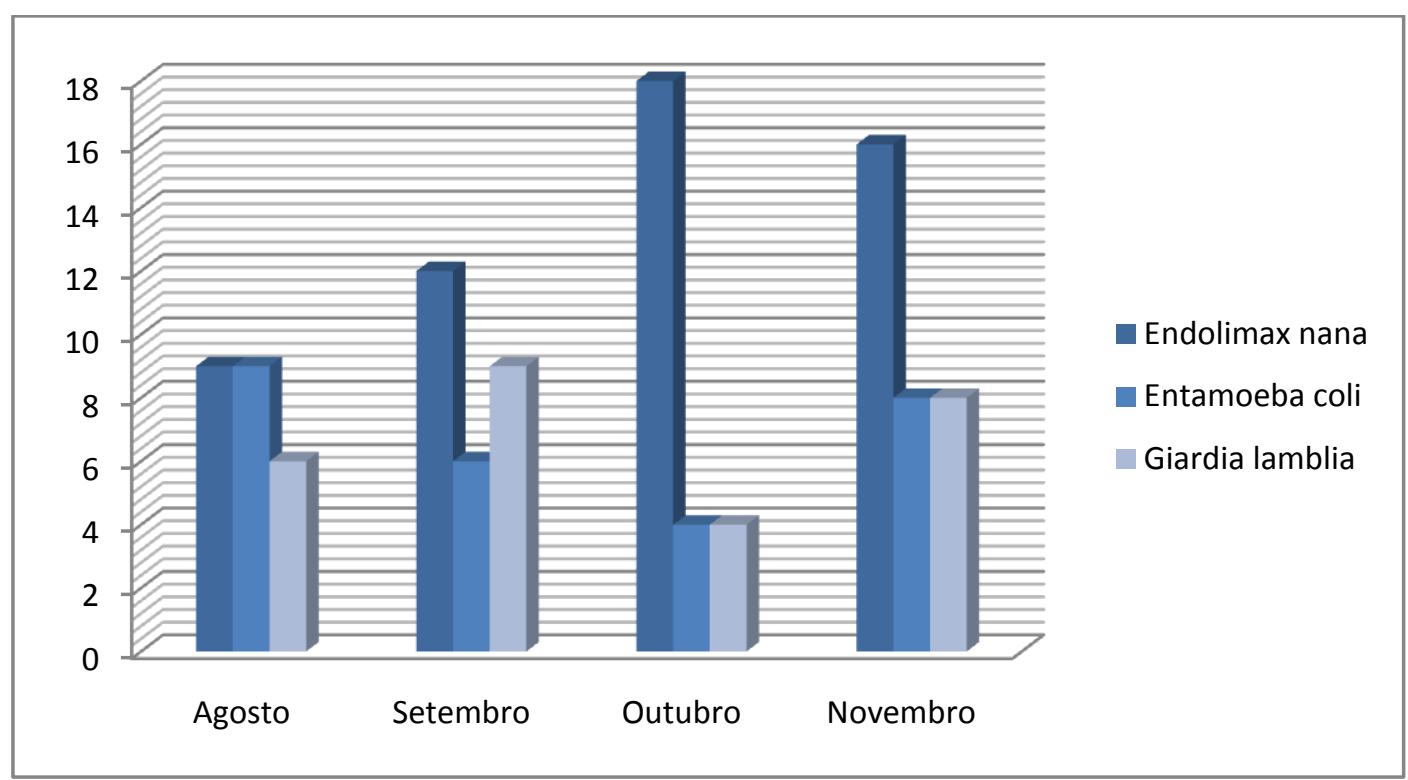

Figura 2 - Prevalência de parasitas encontrados nos meses de Agosto a Setembro na cidade de Morrinhos-GO.

Caso haja diarreia em processos de infestação de giardíase, os trofozoítos serão eliminados nas fezes liquefeitas. Como são sensíveisà dessecação e a agentes físicos e químicos, os trofozoítos são rapidamente destruídos. Portanto,a forma infectante da giárdia são seus cistos.Estes cistos podem se disseminar na poeira, água e através de vetores mecânicos, comomosca e barata, daí contaminando alimentos que podem ser ingeridos, podendo chegar a novos hospedeiros (NEVES, 2005).

O protozoário $G$. lamblia pode originar processos de hipersenibilidade no local parasitado, promovendo a eosinofilia, bem como o aumento de $\operatorname{IgE}$, assim, este parasito pode causar alterações na fisiologia intestinal levando o hospedeiro a desenvolver resposta ao agente agressor (MELO-REIS et al., 2007)
O hábito de viverem bem próximos uns aos outros, dá ao parasito a capacidade de formarum tapete sobre a mucosa intestinal. Este atapetamento, em geral acometendo duodeno e jejuno,prejudica a absorção de nutrientes lipídicos e, consequentemente, os lipossolúveis, uma vez queprovoca a diminuição das microvilosidades do enterócito e impede o contato deste com o nutrienteda luz intestinal. Deste modo, a giárdia impede a absorção de gorduras e vitaminas $\mathrm{A}, \mathrm{D}, \mathrm{E}$ e $\mathrm{K}$,comprometendo a integridade da pele e de seus anexos. A consequência disto é o surgimento demanchas na pele que lembram pano-branco, mas que não descamam ao atrito, enfraquecimentode unhas e queda de cabelo.A não absorção de gorduras leva ao emagrecimento e à presença de fezes gordurosas,amareladas, quase sempre pastosas ou diarreicas, esteatorreicas (esteato $=$ gordura $)($ NEVES, 2005). 


\section{CONCLUSÃO}

A grande prevalência de parasitoses intestinais encontradas nesse tipo de estudo, pode servir de alerta para as autoridades e o poder público, para que medidas urgentes sejam tomadas no sentido da erradicação do problema, ou as altas taxas dos parasitos encontrados podem servir para classificá-los como sendo doenças negligenciadas pela saúde pública e pelos órgãos responsáveis pela formulação de políticas de saúde coletiva nos municípios estudados. $\mathrm{O}$ ideal seria intervenções no sentido de transformar a realidade encontrada nas regiões estudadas, transformando a realidade das populações, visando a melhoria das condições de habitação, saneamento e de higiene, através de medidas sócio-educativas de conscientização da população.

\section{REFERÊNCIAS}

ARAÚJO, A.J.S.; KANAMURA, H.Y.;

DIAS, L.C.S.; GOMES, J.F.; ARAÚJO, S.M.

QuantitativeCoprotest ${ }^{\circledR}$ :

quantificationofhelmintheggs in fecal samplesbycommercialdiagnostic kit. Jornal

Brasileiros de Patologia e Medicina

Laboratorial, Rio de Janeiro, v.39, n.2, p.115-123, 2003.
Através do presente artigo pode-se observar a maior predominância de parasitoses em cidades com menor desenvolvimento socioeconômico, as duas cidades estudadas não apresentam estações de tratamento de esgoto e as águas utilizadas em ambas as cidades são provenientes de mananciais córregos, sujeitos a infestações de parasitos. Os tratamentos de água realizados em ambas as cidades não apresenta qualidade suficiente para eliminação completa dos parasitos. Outro fator a ser observado é a pouca informação da população em relação às parasitoses e os meios mais simples de se evitar a infestação por parte delas. $\mathrm{O}$ alto consumo de hortaliças irrigadas com fontes de água sem tratamento e não higienizadas da maneira correta na região pode estar associado à presença dos parasitos encontrados no presente estudo.

ASTAL Z. Epidemiological survey of the prevalence of parasites among children in Khan Younis governorate, Palestine. Parasitol Res 2004;94:449-51.

CARVALHO, O.S.; GUERRA H.L.;

CAMPOS Y.R.; CALDEIRA R.L. MASSARA C.L. Prevalência de helmintos intestinais em três mesorregiões do Estado de Minas Gerais.Revista da Sociedade Brasileira de Medicina Tropical, Uberaba, MG. v. 35, n.6, p.597-600, 2002. 
CIMERMAN, B.; CIMERMAN, S.

Parasitologia Humana e seus Fundamentos

Gerais. 2.ed. São Paulo: Atheneu, 2001.

FELDMAN M et al. Gastrointestinal and

Liver Disease. Elsevier, 2002.

FERREIRA GR, ANDRADE CFS. Alguns

aspectos socioeconômicos relacionados a

parasitoses intestinais e avaliação de uma

intervenção educativa em escolares de

Estiva Gerbi, SP.

RevSocBrasMedTrop2005;38:402-5.

FERREIRA, H; LALA, ERP; MONTEIRO,

MC; RAIMONDO ML. Estudo

Epidemiológico Localizado da frequiência e

fatores de risco para enteroparasitoese e sua correlação com o estado nutricional de crianças em idade pré-escolar. Publ. UEPG:

Ciências Biológicas. Saúde, Ponta Grossa, 12 (4): 33-40, dez 2006.

GAMBOA MI, BASUALDO JA,

CÓRDOBA MA, PEZZANI BC,

MINVIELLE MC, LAHITTE HB.

Distribution of intestinal parasitoses in relation to environmental and sociocultural parameters in La Plata, Argentina.J

Helminthol2003;77:15-20.

GASPARINI, EA; PORTELLA RB. Manual de Parasitoses Intestinais. Rio de Janeiro: Rubio,2005.
LUDWIG, K. M.; FREI, F.; ALVARES

FILHO, F. \& RIBEIRO-PAES, J. T.,

Correlação entre condições de saneamento

básico e parasitoses intestinais na população de Assis, Estado de São Paulo.

Revista da Sociedade Brasileira de Medicina Tropical, v. 32, n. 5,p. 547-555, set./out., 1999.

MARQUES S.M.T.; BANDEIRA C.; MARINHO DE QUADROS, R. Prevalência de enteroparasitoses em Concórdia, Santa Catarina, Brasil. Parasitologia Latinoamericana. Chile, 60, p.78-81, 2005

MELO-REIS, P.R. Correlação entre eosinofilia e protoparasitose por Giardialamblia em crianças. Revista Brasileira de Análise Clínicas, v.39, n.3, p.237-239, 2007.

MILLER SA, ROSARIO CL, ROJAS E, SCORZA JV. Intestinal parasitic infection and associated symptoms in children attending day care centres in Trujillo, Venezuela. Trop Med Int Health 2003;8:3427.

NEVES, D.P. et al. Parasitologia Humana 11ed. São Paulo: Editora Atheneu, 2005.

NEVES, D.P. Parasitologia Humana. Ed. Guanabara Koogan. 11 ed. 2005. 494p. 
PEDRAZZANI, E. S., MELLO, D. A.,

PIZZIGAT, C. P., PRIPAS, S., FUCCI, M.,

SANTORO, M. C. M. Helmintoses

Intestinais. III- Programa de Educação e

Saúde em Verminose. Revista de Saúde

Publica 23: 189-195. 1989.

PEDROSO, RS; SIQUEIRA, RV. Pesquisa

de cistos de protozoários, larvas e ovos de helmintos em chupetas. Jornal da Pediatria, 73 (1): 21-25, 1997.

REY, L. - Bases da Parasitologia Médica.

Editora Guanabara Koogan, Rio de Janeiro (RJ), 2002.

SALATA, E., CORRÊA, F. M. A., SOGAYAR, R., BARBOSA, M. A. Inquérito Parasitológico na Cecap. Distrito sede de Botucatu, Estado de São Paulo, Brasil. Revista de Saúde Pública 6: 385-392. 1972.

SANTOS, F. L. N.; SOARES, N. M.

Mecanismos fisiopatogênicos e diagnóstico laboratorial da infecção causada pela Entamoebahistolytica. J.Bras. Patol. Med. Lab., v. 44, n. 4, p. 249-261.

SATURNINO, A. C. R. D.; NUNES, J. F. L. \&dSILVA, E. M. A., Relação entre a ocorrência de parasitas intestinais e sintomatologia observada em crianças de uma comunidade carente de Cidade Nova, em Natal - Rio Grande do Norte, Brasil.

Revista Brasileira de Análises Clínicas, v. 35, n.2, p. 82-87, 2003.

SILVA, M.C.M.; MONTEIRO, C.S.P.; ARAÚJO, B.A.V.; SILVA, J.V.; PÓVOA, M.M. Determinação da infecção por Entamoebahistolytica em residentes da área metropolitana de Belém, Pará, Brasil, utilizando ensaio imunoenzimático (ELISA) para detecção de antígenos. Cadernos de Saúde Pública, Rio de Janeiro, v.21, n.3, p.969-973, 2005.

TASHIMA, N.T.; SIMÕES, M.J.S. Parasitas intestinais. Prevalência e correlação com a idade e com os sintomas apresentados de uma população infantil de PresidentePrudente - SP. RBAC, São Paulo, vol.37, n.1, p.35-39, 2005.

TIBIRIÇÁ, S.H.C.; ABRAMO, C.; SIMÕES, A.S.; PINHEIRO, I.O.; RIBEIRO, L.C.; COIMBRA, E.S. Validação do número de lâminas para realização do método de sedimentação espontânea das fezes. HU Revista, Juiz de Fora, v.35, n.2, p.105-110, 2009 .

YERELI K, BALCIOGLU IC, ERTAN P, LIMONCU E, ONAG A. Albendazole as an alternative therapeutic agent for childhood giardiasis in Turkey. ClinMicrobiol Infect 2004;10:527-9. 